\title{
Marciano Barrios Valdés 1926-2015, historiador de la Iglesia
}

\author{
Juan Ricardo Couyoumdjian \\ Roberto Hernández \\ INSTITUTO DE HISTORIA \\ PONTIFICIA UNIVERSIDAD CATÓLICA DE CHILE
}

El fallecimiento de Marciano Barrios Valdés a fines de enero del presente año marca el término de una fructífera vida al servicio de la Iglesia a través de la investigación histórica y de la docencia. Su primera ocupación fue el magisterio. Enseñó sin interrupción durante sesenta y seis años. Su temprana vocación pedagógica la desarrolló en la Congregación de los Hermanos Maristas dando clases en todos los niveles de enseñanza. Esta actividad lo convirtió en un maestro experimentado en el aula y en el trato con sus alumnos. Su actividad comenzó en Lima en 1943. De Perú pasó a Chile y desde 1948 enseñó en diversos establecimientos en Rancagua, Quillota, Curicó, Valparaíso y Santiago. Residiendo en Quillota ingresó a la Universidad Católica de Valparaíso. En 1966 obtuvo el título de Profesor de Historia y Geografía. Al año siguiente el mismo centro de estudios lo tituló Orientador Educacional y, en 1978, Profesor de Religión en Educación Media. Es así como la fe, la ciencia y la educación explican la fecunda vida de trabajo del profesor Barrios que derivó en una sostenida y aguda capacidad para investigar, enseñar y promover el cultivo de la historia eclesiástica. Desde 1966, el aula universitaria, los archivos y las bibliotecas fueron los escenarios que acogieron las inquietudes del maestro historiador Su paso por la Dirección de la Escuela Normal de Varones Maximiano Errázuriz de la Universidad Católica (1968-1971) dejó testimonio de esa maestría y de su capacidad administrativa.

La pasión por la historia de la Iglesia fue refrendada en 1985 por la Universidad de Chile que ese año le otorgó el grado de Doctor en Filosofía con mención en Historia. Diecinueve años antes 
(1966) la Licenciatura en Filosofía y Educación había señalado ese rumbo superior así como la consistencia y el dinamismo de sus numerosas publicaciones. Como profesor de Historia de la Iglesia en la Facultad de Teología de la Pontificia Universidad Católica orientó a numerosas generaciones de estudiantes. Además de sus labores docentes, Marciano Barrios fue Secretario Académico de la $\mathrm{Fa}$ cultad, dirigió la revista Teología y Vida y participó activamente en la publicación de los Anales.

Fue promotor entusiasta de diversas iniciativas intelectuales. Fue el impulsor de la Sociedad de Historia de la Iglesia fundada en 1982 y la Sociedad de Historia de la Educación creada en 1993. Preparó trabajos para las respectivas publicaciones de estos organismos, y acogió con entusiasmo y generosidad a muchos investigadores. La actividad de los años setenta, ochenta y noventa no le impidieron ser miembro activo de la Sociedad Chilena de Historia y Geografía (1982) y del Instituto O'Higginiano (1987). Participó en reuniones científicas, dio conferencias, dirigió memorias y tesis de grado, presentó ponencias en seminarios y congresos, y corrigió centenares de páginas de "pruebas de imprenta”. En medio de estas actividades reunió una biblioteca personal con valioso y escogido material. Junto a los más connotados autores de la historiografía mundial se alineaba una completa bibliografía eclesiástica chilena reunida con predilección de bibliófilo.

Estudió siempre. Con acierto y sazonado fruto buscó y hurgó en los archivos. Sus clases reflejaron esa preparación y su obra impresa da testimonio de ello. Su exposición en el aula no derivaba en una pesada erudición. Era serena, sencilla y equilibrada. Graves asuntos que otrora enfrentaron a la jerarquía o ardorosas controversias entre el poder civil y la Iglesia que prolongaron seculares discordias eran presentadas sin la reactualización odiosa del conflicto sino analizando el arribo a la solución. Un ex alumno dice: "Es evidente su capacidad para tornar sencillo lo complejo, sin que un tratamiento tal conspire a desvalorizar los temas que trata... Su palabra oral tanto como la escrita se modulan y entregan con naturalidad de quien busca comunicar, esclarecer, despertar comprensión admirativa acerca de lo referido, quedándose en un tranquilo y modesto segundo plano"'.

Juan Antonio Massone, "Marciano Barrios Valdés (1926). Lecciones de un historiador de la Iglesia”. Revista de Historia y Geografía. Universidad 
A sus publicaciones monográficas sumó recensiones, informes, bibliografías e índices y redactó crónicas de las actividades de la Sociedad de Historia de la Iglesia. La bibliografía del profesor Barrios tiene un valioso significado heurístico y hermenéutico. Sus trabajos son una fuente ordenada y orientadora. Para los estudiosos de la historiografía eclesiástica chilena es una consulta insoslayable. Su tesis doctoral de 1985 versó sobre "La historiografía eclesiástica chilena escrita por sacerdotes" que fue publicada con ampliaciones en tres sucesivas entregas de los Anales de la Facultad de Teología ${ }^{2}$. Lo anterior fue complementado por un artículo sobre la historia de la Iglesia escrita por laicos, que fue publicado en 1995 La expresión "historiografía eclesiástica", la entendía en toda su amplitud, habiéndose iniciado en el tema con su trabajo sobre la historiografía eclesiástica chilena como instrumento político y la historiografía sobre los seminarios publicados en 1983 y 1984 es decir antes de entregar su tesis No abandonó su interés por el tema y en 1989 escribió acerca de "Algunas tendencias de la historiografía eclesiástica

Católica Silva Henríquez, Santiago, 2006, 277-319, la cita en 280.

2 Sobre estos y demás trabajos suyos véase la bibliografía al final. chilena" con un renovado esfuerzo interpretativo.

Una etapa de madurez, originalidad y sensibilidad del Profesor Barrios Valdés está señalada por las sugerentes incursiones en las formas o manifestaciones de piedad católica situadas en su atmósfera social, temporal y pastoral. El primer trabajo fue La espiritualidad en tiempos de Santa Teresa de Los Andes (1994). Le siguieron La espiritualidad en tiempos del Padre Alberto Hurtado 1931-1961 (1995) y La espiritualidad en tiempos del Vaticano II (1996). Estos tres trabajos sobre conductas orientadas por la fe y que forman un conjunto, fueron precedidos en 1987 por "La religiosidad popular en Chile" y en 1989 por un estudio sobre "Las raíces de la devoción mariana en Chile colonial".

Su interés por la historia eclesiástica dio origen a numerosas aportes al conocimiento de instituciones religiosas, como son las órdenes de Santo Domingo y de San Francisco, la Compañía de Jesús, el seminario de los Santos Ángeles Custodios, la Facultad de Teología de la Universidad Católica y los Hermanos Maristas. Una visión general la encontramos en: "La Iglesia en Chile. Sinopsis histórica" (1987) y "Chile y su Iglesia: una sola historia" (1992). Los 
libros del profesor Barrios Valdés no son extensos. La excepción son las 380 páginas de "La presencia franciscana en Chile. Sinopsis histórica 1553-2003" investigación encargada por la Orden Seráfica y publicada el 2003 con motivo de celebrarse los 450 años de su apostolado en Chile. Fue escrita consultando fuentes directas, compulsando el archivo de la orden, lo que le permitió originales hallazgos.

A todo lo anterior se suman sus numerosas reseñas biográficas de miembros del clero, En el ámbito de la prosopografía cabe destacar la revisión, ordenamiento y publicación del Diccionario Biográfico del clero secular chileno 1918-1969 del presbítero Raimundo Arancibia Salcedo quien confió su manuscrito a las manos organizadoras del profesor Barrios, a lo que agregó un complemento publicado en Teología y Vida bajo el nombre del P. Arancibia ${ }^{3}$. Otro encargo fue el que asumió a petición de monseñor Carlos Oviedo Cavada para la edición del Episcopologio chileno, cuyos cuatro volúmenes con sus

3 Raimundo Arancibia Salcedo, Diccionario biográfico del clero secular de Chile. 1918-1969, Editorial Neupert, Santiago, 1969; del mismo "Diccionario biográfico del clero secular chileno 1969-1982. Anotaciones al diccionario de L. F. Prieto del Río" Teología y vida, 23/4 (2002), pp. 278-344. dos mil páginas en total se publicaron en 1992. El profesor Barrios orientó las bibliografías de los trabajos de los algunos de los participantes en el proyecto ${ }^{4}$.

El año 2009 fue su último con actividad docente. En marzo del 2010 informó los seminarios que tenía pendientes. La actividad incesante se interrumpió. La salud se había resentido y el malestar fue progresivo. Dos legajos con apuntes sobre la Compañía de Jesús y los Hermanos Maristas quedaron inconclusos. Donó la mayor parte de su biblioteca personal.

El profesor Marciano Barrios se entregó sin límite al trabajo académico y a la investigación. Su vida fue un magisterio generoso y su obra impresa un legado valioso y vigente. Fue un hombre justo y un cristiano de ciencia y conciencia.

Bibliografía de Marciano

BARRIOS VALDÉS

La presente bibliografía de Marciano Barrios ha sido elaborada a partir del "Fichero bibliográfico" de la revista Historia de la

\footnotetext{
Carlos Oviedo Cavada (director)Marciano Barrios, (editor), Episcopologio Chileno 1561-1815, dirigido por Carlos Oviedo C. y editado por Marciano Barrios, Editorial Universidad Católica de Chile, Santiago, 1992, 4 volúmenes
} 
Universidad Católica; del trabajo de Juan Antonio Massone ya citado; del libro de Cristián Gazmuri sobre historiografía chilena ${ }^{5}$, del Episcopologio Chileno 1561-1815 mencionado más arriba y del currículo y bibliografía autógrafa del Prof. Marciano Barrios V.

Las referencias están ordenadas por materia en cinco secciones como sigue: I.- Historia de la Iglesia; II.- Historiografía eclesiástica, III.- Semblanzas biográficas de eclesiásticos, IV.- Educación y textos escolares y V.- Historia general y otros temas. No se han incluido las recensiones de libros y revistas, que alcanzan a 256 , según lo contabilizado por el profesor Massone, ni las reseñas de actividades de la Sociedad de Historia de la Iglesia en Chile, aparecidas en los sucesivos números del Anuario de Historia de la Iglesia en Chile desde sus inicios en 1983 hasta el número 22 del año 2004.

Se han empleado las siguientes abreviaturas

AFT

Anales de la Facultad de Teología.

Cristián Gazmuri R., La historiografía chilena, tomo II (1920-1970). Centro de Investigaciones Diego Barros Arana, Taurus, Santiago, 2009.
$\mathrm{AHICh}$

Anuario de Historia de la Iglesia en Chile

BHG

Boletin de Historia y Geografía. Instituto Profesional de Estudios Superiores Blas Cañas, luego Universidad Católica Blas Cañas, luego Universidad Católica Silva Henríquez

Episcopologio Episcopologio Chileno 1561-1815, dirigido por Carlos Oviedo Cavada y editado por Marciano Barrios, Santiago, Editorial Universidad Católica de Chile, 1992, 4 volúmenes,

$\mathrm{RC}$ La Revista Católica.

TV Teología y Vida.

\section{Historia de la Iglesia}

1. "La religiosidad popular en Chile". Segundo encuentro de religiosidad popular. Historia y Misión. Ponencias, aportes y experiencias. Santiago, mayo 1977. Ediciones Mundo, Santiago, 1977, 26-40.

2. "La religiosidad popular en Chile. Intento de periodificación", $T V$ Vol. XVII, $\mathrm{N}^{\circ}$ 2-3, 1977, 129-144. 
3. "Breve Historia de la Iglesia en Latinoamérica”. ONAC, 1983, 159-185.

4. "Prólogo" en Raimundo Ghigliazza O.P. Historia de la Provincia Dominicana en Chile Tomo II, Salesianos, Santiago, 1985, 5-9.

5. "La Iglesia en Iberoamérica. Luces y sombras", $R C \mathrm{~N}^{\circ} 1.068,1985$, 113-122.

6. "Los Dominicos en la evangelización de Chile", en Quienes son los Dominicos hoy. Colección Cuarto Centenario Dominicos. Santiago 1986, 41-54.

7. "Condiciones de la evangelización en América española”. $R C \mathrm{~N}^{\circ}$ 1.072, 1986, 273-280.

8. "La religiosidad popular en Chile" TV, Vol. XXVIII, $\mathrm{N}^{\circ} 1-2,1987$, 85-93.

9. La Iglesia en Chile. Sinopsis histórica. Ediciones Pedagógicas Chilenas, Santiago, 1987.

10. "Raíces de la devoción mariana en Chile Colonial". $R C \mathrm{~N}^{\circ} 1.082$, 1989, 97-105.

11. "La Iglesia en América Latina durante el siglo XX". TV, Vol. XXX, $\mathrm{N}^{\circ} 4,1989,299-310$.

12. "Raíces cristianas de la cultura chilena”, en Laicado Iglesia Cultura y Sociedad, I.P.E.S. Blas Cañas, Centro Teológico Filosófico, Santiago, 1989 15-28.

13. "El Papa Paulo VI y la prensa chilena”. Colloquio Internazionale di Studio. Paulo VI e i problemi eclesiologici al Concilio, Edizioni Studium-Roma, Brescia, 1989.
14. "La Facultad de Teología de la Pontificia Universidad Católica de Chile. Algo de su historia, 19351988" (Primera Parte). AHICh $\mathrm{N}^{\circ} 9$ 1991, 105-131.

15. "La Independencia y su gravitación en la evangelización". TV, Vol. XXXII 1991, 111-124.

16. "La Iglesia durante los siglos XIX y XX". BHG No 8, 1991, 53-60.

17. Chile y su Iglesia: una sola historia. Prólogo de Mons. Carlos Oviedo Cavada. Editorial Salesiana, Santiago 1992,

18. "La primera evangelización en Chile", Actas del Séptimo Simposio de Teología Histórica. Valencia, 1992, 243-255.

19. "La Facultad de Teología de la Pontificia Universidad Católica de Chile. Algo de su historia 19351988" (Segunda Parte). AHICh $\mathrm{N}^{\circ} 10,1992$ 139-158.

20. "La Iglesia en el Nuevo Mundo". El Mercurio. Ediciones Especiales. Quinto Centenario. Santiago 12 de septiembre de 1992.

21. "Los jesuitas en la Facultad de Teología”. Mensaje $\mathrm{N}^{\circ} 420$, julio 1993, 293-296.

22. "La Facultad de Teología de la Pontificia Universidad Católica de Chile. Algo de sus historia 19351988 (Continuación de la segunda parte)". AHICh N 11, 1993 , 129-148.

23. "Espiritualidad en Chile en los años 1860-1930" en Concepto de santidad, significado y proyecciones: el caso de Santa Teresa de Los An- 
des, editor Roberto Vega Masso. Universidad Diego Portales, Santiago, 1993, pp. 8-19.

24. "Religión mapuche y Cristianismo: un encuentro". $T V$ Vol. $\mathrm{N}^{\circ}$ XXXV, 1994, 297-306.

25. La espiritualidad chilena en tiempos de Santa Teresa de Los Andes. San Pablo ediciones, Santiago 1994.

26. "La Facultad de Teología de la Pontificia Universidad Católica de Chile 1935-1988. Algo de su historia”. (Tercera Parte y Epílogo) AHICh No 12, 1994, 153-189.

27. La espiritualidad en los tiempos del Padre Hurtado, 1931-1961. Universidad Católica Blas Cañas. Serie de Investigaciones 7 , Santiago, 1995.

28. "La espiritualidad chilena en tiempos del Padre Hurtado". RC $\mathrm{N}^{\circ} 1.105,1995,21-29$.

29. La Facultad de Teología de la Pontificia Universidad Católica de Chile. Sesenta años de historia al servicio de Chile y de su Iglesia (1935-1995). Ediciones Sociedad de Historia de la Iglesia en Chile, Santiago, 1995.

30. La espiritualidad católica chilena en tiempos de Vaticano II. Universidad Católica Blas Cañas. Serie Investigaciones 10, Santiago, 1996.

31. "El regalismo y la romanización de la Iglesia en Chile", AHICh $\mathrm{N}^{\circ} 18$, 2000, 77-87.

32. "Algunas notas sobre las misiones franciscanas en la Araucanía. Si- glo XIX". AHICh N $\mathrm{N}^{\circ} 20,2002$, 63-74.

33. "Relaciones entre Iglesia y Estado". $B H G \mathrm{~N}^{\circ} 17,2002,151-163$.

34. Presencia Franciscana en Chile Sinopsis Histórica 1553-2003. Publicaciones del Archivo Franciscano, Santiago 2003.

35. "Aporte franciscano a la educación en Chile". Revista de Historia y Geografia No 17 Universidad Católica C. Raúl Silva Henríquez, 2003, 53-67.

36. "Un aporte italiano a la Iglesia chilena en el siglo XX", en Los Franciscanos en Chile. Una historia de 450 años, René Millar y H. Aránguiz editores, Academia Chilena de la Historia. Santiago, 2005, 317-334.

37. "Arte y congregaciones religiosas en Chile". Testimonio $\mathrm{N}^{\circ} 215$. Mayo Junio 2006, 53-61.

\section{Historiografía ECLESIÁSTICA}

1. Teología y Vida. Índice general de los volúmenes de I- XX, 19601979, Teología y Vida, Santiago, 1981.

2. "Bibliografía sumaria del Profesor Julio Jiménez". AFT Vol. XXXIII, 1982, 15-23.

3. "Historiografía eclesiástica chilena como instrumento político", AHICh $\mathrm{N}^{\circ} 1,1983,11-26$.

4. La historiografía sobre los seminarios". AHICh No 2, 1984, 61-75.

5. La historiografía eclesiástica sobre la Independencia”. Ciclos de Con- 
ferencias 1983-1984. Santiago, Universidad de Santiago, 1984, 43-52.

6. "Las parroquias chilenas en la historiografía", AHICh, N 3, 1985, 165-177.

7. "Tendencias de la historiografía eclesiástica chilena durante el siglo XIX (1848-1918)", TV Vol., XXVII, N² 2-3, 1986, 191-205.

8. "Pensamiento teológico en Chile. Contribución a su estudio III. Historiografía eclesiástica chilena 1848-1918”. AFT Vol. XXXVIII, 1987, 1-171.

9. "Algunas tendencias de la historiografía eclesiástica chilena." AHICh $\mathrm{N}^{\circ} 7,1989,217-229$.

10. "Pensamiento Teológico en Chile. Contribución a su estudio. IV. Historiografía eclesiástica chilena 1918-1988”. AFT Vol. XL, 1989, 1-110.

11. "Historiografía eclesiástica 1848 1988. La Iglesia: una visión de los laicos". Historia $\mathrm{N}^{\circ} 28,1994$, 5-75.

12. "Pensamiento Teológico en Chile. Contribución a su estudio. VI La Iglesia en la Historiografía de los civiles 1848-1988". AFT Vol. XLVI, 1995, 1-127.

13. "Revistas teológicas chilenas". AHICh $\mathrm{N}^{\circ} 18,2000$, pp. 349-359.

14. "Historiadores y cronistas de la Orden de los Hermanos Menores en Chile". Revista de Historia y Geografia No 18 . Universidad
Católica C. Raúl Silva Henríquez, 2004, 9-32.

III. Semblanzas Biográficas DE ECLESIÁSTICOS

1. "José Ignacio Cienfuegos Arteaga. Ardoroso patriota y virtuoso eclesiástico". Ciclo de Conferencias Universidad de Santiago de Chile, Santiago, 1982, 80-87.

2. "Carlos Silva Cotapos. Historiador de la Iglesia en Chile". AFT Vol. XXXIII, 1982, 271-288.

3. "Pensamiento historiográfico de Gabriel Guarda. Premio Nacional de Historia”. Dimensión Histórica de Chile. Universidad Metropolitana de Ciencias de la Educación. Vol. 4-5, 1987-1988, 187-203.

4. “José Ignacio Cienfuegos. Sacerdote ilustrado y Obispo de Concepción”. AFT Vol. XXXIX, 1988, 295-329.

5. "Pedro de Azuaga", Episcopologio, t I, 1992, 151-156.

6. "Francisco González de Salcedo". Episcopologio, t I, 1992, 227-246.

7. "Antonio de San Miguel". Episcopologio t. III, 1992, 329-362.

8. "Agustín de Cisneros." Episcopologio t. III, 1992, 363-374.

9. "Miguel Antonio de Argandoña". Episcopologio t IV, 1992, 593-597.

10. "Crescente Errázuriz. Periodista e historiador". $B H G \mathrm{~N}^{\circ} 10,1993$, 191-198.

11. "Figuras de proa de la historia agustiniana de Chile". AHICh $\mathrm{N}^{\circ}$ 13, 1995, 55-65. 
12. "Walter Hanisch Espíndola. Premio Nacional de Historia”. TV Vol. XXXVII, 1996, 275-278.

13. "Navegante de mares ignotos". AHICh, No 14, 1996, pp. 9-15. (referido a W. Hanisch).

14. "Fidel Araneda Bravo, sacerdote, periodista, historiador". $B H G \mathrm{~N}^{\circ}$ 13, 1997, 5-17.

15. "Fernando Cifuentes Grez. Sacerdote y Educador”. $R C \mathrm{~N}^{\circ} 1.115$, 1997, 209-213.

16. "Joaquín Larraín Gandarillas. Humanista y Educador". $R C \mathrm{~N}^{\circ}$ 1.116, 1997, 315-320.

17. "Carlos Casanueva Opazo Apóstol Social”. $R C \mathrm{~N}^{\circ} 1.118,1997$, pp. 134-139.

18. "José Ignacio Cienfuegos y Manuel Vicuña, paralelismo y convergencia”. AHICh, No 15. 1997 , 101-112.

19. "Oscar Larson Soudy (18931974)”. RC N $\mathrm{N}^{\circ} 1.119,1998$, 306-311.

20. "Jorge Gómez Ugarte. Educador." $R C \mathrm{~N}^{\circ} 1.120,1998,306-311$.

21. "Roberto Polain y su ideario pedagógico”. $R C \mathrm{~N}^{\circ} 1.121,1999$, 53-58.

22. "Carlos Oviedo Cavada. Historiador de la Iglesia”. $R C \mathrm{~N}^{\circ} 1.122$, 1999, 131-137.

23. "Raúl Silva Henríquez. Educador". $R C \mathrm{~N}^{\circ} 1.123,1999$, 236-243.

24. "Rubén Castro Rojas. Su obra educacional.” $R C \mathrm{~N}^{\circ}$ 1.124, 338-345.

25. "El Cardenal Raúl Silva Henríquez y su aporte a la educación chilena”. AHICh No 18, 2000, 145-156.

26. "Aporte de tres sacerdotes seculares a la educación en Chile". Revista de Historia de la Educación Vol. IV, Santiago, 2000, 81-93.

27. "Manuel Larraín Errázuriz y su época”, $R C \mathrm{~N}^{\circ} 1.125,2000$, 60-67.

28. "Luis Francisco Prieto del Río. Bibliógrafo e Historiador". $R C \mathrm{~N}^{\circ}$ 1.127, 2000, 227-240.

29. "Emilio Vaisse. Apóstol y periodista”. $R C \mathrm{~N}^{\circ}$ 1.128, 2000, 437-444.

30. "Walter Hanisch, SJ. In memoriam 26-8-1916- 13-10-2001". AHICh $\mathrm{N}^{\circ}$ 19, 2001, 9-13.

31. "Maximiano Arias Reyero. Fundador de SELADOC". $R C, \mathrm{~N}^{\circ}$ 1.129, 2001, 42-52.

32. "Gilberto Fuenzalida Guzmán. Pedagogo y Catequista”. $R C \mathrm{~N}^{\circ}$ 1.130, 2001, 117-127.

33. "Presbítero Humberto Muñoz Ramírez. Cooperativismo, ecumenismo y campesinado", $R C \mathrm{~N}^{\circ}$ 1.131, 2001, 233-240.

34. "Monseñor Reinaldo Muñoz Olave. Historiador de la Diócesis de Concepción”. $R C \mathrm{~N}^{\circ} 1.132$, 2001, 321-325.

35. "Presbítero Elías Lizana Martínez. Archivero de Arzobispado de Santiago de Chile 1911-1919". RC $\mathrm{N}^{\circ} 1.133,2002,70-80$.

36. Monseñor Francisco Vives Estévez". $R C \mathrm{~N}^{\circ}$ 1.134, 2002, 151-157.

37. "Juan Ignacio González Eyzaguirre. El Arzobispo de los obreros”. RC No 1.135, 2002, 245-251. 
38. "Fray Luis Toloza Olivares Molina”. $R C \mathrm{~N}^{\circ} 1.138,2003,147-153$.

39. Monseñor Bernardino Berríos Gainza. RC N 1.138, 44-51.

40. "Notas en torno a las actividades pastorales del Cardenal Raúl Silva Henríquez". Revista de Ciencias Religiosas. Universidad Raúl Silva Henríquez, Santiago, Vol. XI, 2003, 37-45.

41. "Dos generaciones de intelectuales cristianos 1900-1960". AHICh, $\mathrm{N}^{\circ} 21,2003,41-48$.

42. "Joaquín Larraín Gandarillas. Una figura de proa en la educación chilena." Pensamiento Educativo. Pontificia Universidad Católica de Chile, Santiago, Vol. 34, 2004, 255-270.

43. "Fray Antonio Pérez de Maldonado". $R C \mathrm{~N}^{\circ} 1.141,2004$, 49-55.

44. "Rafael Larraín Errázuriz". $R C \mathrm{~N}^{\circ}$ 1.148, 2005, 317-323.

\section{EDUCACIÓN Y TEXTOS ESCOLARES}

1. Documentos para la Historia Moderna. Ministerio de Educación. Centro de Perfeccionamiento del Magisterio, Santiago, 1980, (mimeografiada).

2. Manual de Historia Moderna y Contemporánea. Ediciones pedagógicas, Librería Francesa, Santiago, 1983.

3. Historia y Geografia II. Segundo año de Educación Media. Ediciones Pedagógicas. Librería Francesa, Santiago, 1984.

4. "Manuales Universitarios de Historia Moderna”. $B H G \mathrm{~N}^{\circ} 2,1986$, 15-17.
5. "El documento en la enseñanza de la Historia.” $B H G, \mathrm{~N}^{\circ}$ 5, 1988, 2-7.

6. Época Contemporánea. Manual de Historia Universal. Editorial Universitaria. Santiago, 1989.

7. "Una tradición pedagógica. Los Hermanos Maristas." Revista de Pedagogía. FIDE Secundaria, $N^{\circ}$ 361, Santiago, 1993, 283-288.

8. "Pragmatismo e idealismo en educación”. BHG, $\mathrm{N}^{\circ}$ 11, 1994, 131-142.

9. “Utopías." Revista de Historia de la Educación, Vol. I, 1995, 7-8.

10. "La educación durante la Colonia". Revista de Historia de la Educación Vol. I, 1995, 119-126.

11. "Modernización educacional". Revista de Historia de la Educación. Vol. II, 1996, 5-6.

12. "Reflexiones en torno a la Reforma Educacional de 1965”, Foro Educacional $\mathrm{N}^{\circ}$ 1. Universidad Católica Blas Cañas, Santiago, 1996, pp. 49-62.

13. "La novedad del pasado". Revista de Historia de la Educación Vol. III, 1997, 9-10.

14. "Montaigne y la educación moderna”. Revista de Historia de la Educación, Vol. III, 1997, 39-48.

15. "Los objetivos transversales de los programas de educación”. Foro Educacional. Universidad Católica Blas Cañas, Vol. 2, Santiago, 1997, 83-90.

16. "Las clases de religión y las reformas educacionales del siglo XX". AHICh. No 17, 1999, 187-194. 
17. "Adelante". Revista de Historia de la Educación $\mathrm{N}^{\circ}$ 4, Santiago, 2000, 7-8.

18. "La enseñanza de la Historia”. $B H G \quad \mathrm{~N}^{\circ} 15,2000-2001$, 275-327.

\section{Historia GENERAL y OtROS TEMAS}

1. "Las enseñanzas de otro momento difícil: la caída de Roma". TVVol. IX, N 3-4, 1968, 187-196.

2. "Veinte siglos de acción social". Ediciones Mundo, Santiago, 1976.

3. "Richelieu y la formación de los Estados Modernos". El Mercurio, Santiago 28-8-1977.

4. "Fe y cultura durante el siglo XVI". TV Vol. XIX, 1978, 100-110.

5. "Dos notas en torno al Barroco". TV Vol. XXVI, Nº 1, 1986, 81-87.

6. "El pensamiento histórico de Fernand Braudel" Revista de Historia Universal. Instituto de Historia. Pontificia Universidad Católica de Chile. N VI, Santiago, 1986, 165-175.
7. "Tres hombres del Renacimiento y sus ideas". BHG $\mathrm{N}^{\circ} 4,1987$, 9-15.

8. "La economía carolingia". $B H G$ $\mathrm{N}^{\circ}$ 5, 1989, 10-17.

9. "Mariano Picón Salas. Una visión historiográfica sobre Hispanoamérica". Lengua, literatura e identidad. Ediciones Piedrazul, Santiago, 1991, 180-189.

10. "A propósito de don Antonio García y su Historia de Chile". Mapocho, № 35, 1994, 181-192.

11. "Encarnación y trascendencia, cruzada y misión”. BHG, $\mathrm{N}^{\circ} 12$. 1996, 245-260.

12. "Tolerancia e intolerancia e intolerancia en la Edad Media. Ideas y hechos". BHG, $\mathrm{N}^{\circ} 14,1998$, 357-364.

13. "El Barroco. Sello del pueblo chileno". AHICh No 16, 1998, 139-148.

14. "Universidad y Humanismo. Cardenal Raúl Silva Henríquez". Boletin de Filosofia $\mathrm{N}^{\circ} 10$. Universidad Católica Raúl Silva Henríquez, 1999, 209-218. 
